\title{
DETERMINAÇÃo ANALÍTICA DA INDICATRIZ ÓTICA DE MINERAIS*
}

\author{
IBRAHIM OCTAVIO ABRAHÃO** \\ ARARY MARCONI **
}

\begin{abstract}
RESUMO
Dado um mineral em seç̧ão delgada arbitrária, na platina universal, estuda-se a possibilidade teórica de determinar os índices de refração principais mediante medições de extinção e de birrefringência, por via analítica. Demonstra-se que, teoricamente, é possível determinar $\mathrm{Np}, \mathrm{Nm}$ e $\mathrm{Ng}$ e, independentemente, $\mathrm{B}=\mathrm{Ng}-\mathrm{Np}$.
\end{abstract}

\section{INTRODUÇÃO}

O mé todo analítico permite determinar a orientação da indicatriz ótica de um mineral, dado em secção delgada, mediante simples medições de ângulos de extinção. Quanto à determinação analítica da grandeza da indicatriz (cálculo de $\mathrm{Np}, \mathrm{Nm} \mathrm{e} \mathrm{Ng}$ ), $\mathrm{CHO}$ MARD (1934) a condiciona à prévia medição de Nm.

Estuda-se, neste trabalho, de um ponto de vista teórico, a possibilidade de determinação analítica dos índices de refração principais, sem a medição de $\mathrm{Nm}$. Essa possibilidade teórica, já mencionada por ABRAHÃO (1968) é estudada com e sem a associação de medições de birrefringência às de ângulos de extinção. $O$ obje tivo é mostrar que, teoricamente, é possível obter $\mathrm{Np}, \mathrm{Nm}$ e $\mathrm{Ng}$ por via analítica, empregando-se operações de extinção e birrefringência e o valor $\mathrm{B}=\mathrm{Ng}-\mathrm{Np}$, também calculado analiticamente.

\section{REVISÃO DE LITERATURA}

CHOMARD (1934), desenvolveu as bases teóricas do método analítico, cuja marcha de cálculo conduz à determinação, em orientação e grandeza, da indicatriz ótica de um mineral dado em seç̧ão delgada arbitrária. A orientação da indicatriz é obtida a partir de pelo menos três operações de extinção $(\varphi, \theta, \Psi)$, executadas na platina universal. Para a determinação da grandeza da indicatriz, CHOMARD associa medições de birrefringência às de extinção. Além disso, introduz o valor de $\mathrm{Nm}$ obtido pelo método do duque de Chaulnes. Na determinação da grandeza, portanto, o método é apenas parcialmente analítico, uma vez que emprega um valor medido de $\mathrm{Nm}$ no cálculo de $\mathrm{Np}$ e $\mathrm{Ng}$.

CHOMARD faz menção à possibilidade de uma solução inteiramente analítica, mediante o emprego da técnica de aproximações sucessivas para o cálculo de $\mathrm{Nm}$. Em todos os exemplos sobre a aplicação do método, todavia, utiliza o valor medido de $\mathrm{Nm}$.

ABRAHÃO (1968), aplica o método de maneira sistemática a plagioclásios, mediante um programa para computador que torna desprezivel a dificuldade imposta pela mar-

\footnotetext{
* Enviado para publicação em 23/5/1975.

* Departamento de Solos e Geologia, ESALQ - USP.
} 
cha de cálculo. Menciona a possbilidade de uma solução analítica para o problema, dispensando a medição de $\mathrm{Nm}$. Mostra que com n operações de extinção e birrefringência $(\varphi, \theta, \Psi, \mathrm{r})$ é possivel determinar $\mu$ e $\lambda$, que levam ao completo conhecimento do elipsóide. A guisa de exemplo, desenvolve os determinantes para 3 operações $(\varphi, \theta, \Psi, r)$.

ABRAHĀO, todavia, não cogita de determinar se o valor calculado para $\mu=\mathrm{Nm}$ é, verdadeiramente, um índice de refração ou o semi-eixo de um dos infinitos elipsóides de mesmo $2 \mathrm{~V}$.

ABRAHÃO e GODOY (1971), publicam o programa para computador eletrônico que torna o método analítico exeqüível na prática. Fornecidas três operações de extinção $(\varphi, \theta, \Psi)$, o computador fornece os ângulos diretores dos eixos óticos $A_{1}$ e $A_{2}, o$ valor do ângulo $2 \mathrm{~V}$, precedido de sinal que indica se emerge na lâmina a bissetriz aguda ou obtusa e $\mathrm{C}=\mu \lambda / \mathrm{r}$, valor que dá o sinal ótico do mineral.

\section{DE TERMINAÇÃO ANALITICA DE $\mu=\mathrm{Nm}$}

\section{Emprego da equação geral de extinção e birrefringência}

No desenvolvimento do método analítico, chega-se à expressão seguinte, a que devem obedecer todas as operações de extinção e de birrefringência $(\varphi, \theta, \Psi, \mathrm{r})$ :

$$
\frac{r}{\mu}=2 \lambda(\alpha-\beta)+6 \lambda^{2}\left(\alpha^{2}-\beta^{2}\right)+20 \lambda^{3}\left(\alpha 3-\beta^{3}\right)+\ldots
$$

em que :

$$
\begin{aligned}
& \alpha=a^{2} j j^{\prime}+a^{\prime 2} m m^{\prime}+a^{\prime \prime 2} n n^{\prime}+a^{\prime} a^{\prime \prime}\left(m n^{\prime}+m^{\prime} n\right)+a a^{\prime \prime}\left(j n '+j{ }^{\prime} n\right)+a a^{\prime}\left(j m^{\prime}+j ' m\right) \\
& \beta=b^{2} j j^{\prime}+b^{\prime 2} m m^{\prime}+b^{\prime \prime 2} n n^{\prime}+b^{\prime} b^{\prime \prime}\left(m n^{\prime}+m^{\prime} n\right)+b b^{\prime \prime}\left(j n^{\prime}+j^{\prime} n\right)+b b^{\prime}\left(j m^{\prime}+j{ }^{\prime} m\right)
\end{aligned}
$$

$\mathrm{j}, \mathrm{m}, \mathrm{n}=$ co-senos diretores do eixo óticc $A$,

j', m', n' = co-senos dire tores do eixo ótico $\mathrm{A}_{2}$

$$
\begin{aligned}
& \mathrm{a}=\cos \varphi \cdot \cos \Psi-\operatorname{sen} \varphi \cdot \operatorname{sen} \Psi \cdot \cos \theta \\
& a^{\prime}=-\cos \Psi \cdot \operatorname{sen} \varphi-\operatorname{sen} \Psi \cdot \cos \varphi \cdot \cos \theta \\
& a^{\prime \prime}=\operatorname{sen} \Psi \cdot \operatorname{sen} \theta \\
& \mathrm{b}=-\cos \varphi \cdot \operatorname{sen} \Psi-\operatorname{sen} \varphi \cdot \cos \Psi \cdot \cos \theta \\
& b^{\prime}=-\operatorname{sen} \varphi \cdot \operatorname{sen} \Psi+\cos \varphi \cdot \cos \Psi \cdot \cos \theta \\
& b^{\prime \prime}=-\cos \Psi \cdot \operatorname{sen} \theta
\end{aligned}
$$

É possível determinar analiticamente $\mu$ e $\lambda$ a partir de $n$ operações de extinção e birrefringência, que conduzem a $n$ expressões (1). Tomemos três: $\left(\varphi_{1}, \theta_{1}, \Psi_{1}, r_{1}\right)$, $\left(\varphi_{2}, \theta_{2}, \Psi_{2}, r_{2}\right)$ e $\left(\varphi_{3}, \theta_{3}, \Psi_{3}, r_{3}\right)$. Obtém-se: 


$$
\begin{aligned}
& \mathrm{r}_{1}=2 \mu \lambda\left(\alpha_{1}-\beta_{1}\right)+6 \mu \lambda^{2}\left(\alpha_{1}^{2}-\beta_{1}^{2}\right)+20 \mu \lambda^{3}\left(\alpha_{1}^{3}-\beta_{1}^{3}\right) \\
& \mathrm{r}_{2}=2 \mu \lambda\left(\alpha_{2}-\beta_{2}\right)+6 \mu \lambda^{2}\left(\alpha_{2}^{2}-\beta_{2}^{2}\right)+20 \mu \lambda^{3}\left(\alpha_{2}^{3}-\beta_{2}^{3}\right) \\
& \mathrm{r}_{3}=2 \mu \lambda\left(\alpha_{3}-\beta_{3}\right)+6 \mu \lambda^{2}\left(\alpha_{3}^{2}-\beta_{3}^{2}\right)+20 \mu \lambda^{3}\left(\alpha_{3}^{3}-\beta_{3}^{3}\right)
\end{aligned}
$$

Portanto, sendo:

$$
\Delta^{\prime}=\left|\begin{array}{lll}
\mathrm{r}_{1} & 6\left(\alpha_{1}^{2}-\beta_{1}^{2}\right) & 20\left(\alpha_{1}^{3}-\beta_{1}^{3}\right) \\
\mathrm{r}_{2} & 6\left(\alpha_{2}^{2}-\beta_{2}^{2}\right) & 20\left(\alpha_{2}^{3}-\beta_{2}^{3}\right) \\
\mathrm{r}_{3} & 6\left(\alpha_{3}^{2}-\beta_{3}^{2}\right) & 20\left(\alpha_{3}^{3}-\beta_{3}^{3}\right)
\end{array}\right|
$$

$$
\Delta "=\left|\begin{array}{lll}
2\left(\alpha_{1}-\beta_{1}\right) & r_{1} & 20\left(\alpha_{1}^{3}-\beta_{1}^{3}\right) \\
2\left(\alpha_{2}-\beta_{2}\right) & r_{2} & 20\left(\alpha_{2}^{3}-\beta_{2}^{3}\right) \\
2\left(\alpha_{3}-\beta_{3}\right) & r_{3} & 20\left(\alpha_{3}^{3}-\beta_{3}^{3}\right)
\end{array}\right|
$$

$$
\Delta^{\prime \prime}=\left|\begin{array}{lll}
2\left(\alpha_{1}-\beta_{1}\right) & 6\left(\alpha_{1}^{2}-\beta_{1}^{2}\right) & \mathrm{r}_{1} \\
2\left(\alpha_{2}-\beta_{2}\right) & 6\left(\alpha_{2}^{2}-\beta_{2}^{2}\right) & \mathrm{r}_{2} \\
2\left(\alpha_{3}-\beta_{3}\right) & 6\left(\alpha_{3}^{2}-\beta_{3}^{2}\right) & \mathrm{r}_{3}
\end{array}\right|
$$

$$
\Delta=\left|\begin{array}{lll}
2\left(\alpha_{1}-\beta_{1}\right) & 6\left(\alpha_{1}^{2}-\beta_{1}^{2}\right) & 20\left(\alpha_{1}^{3}-\beta_{1}^{3}\right) \\
2\left(\alpha_{2}-\beta_{2}\right) & 6\left(\alpha_{2}^{2}-\beta_{2}^{2}\right) & 20\left(\alpha_{2}^{3}-\beta_{2}^{3}\right) \\
2\left(\alpha_{3}-\beta_{3}\right) & 6\left(\alpha_{3}^{2}-\beta_{3}^{2}\right) & 20\left(\alpha_{3}^{3}-\beta_{3}^{3}\right)
\end{array}\right|
$$

obtém-se :

$$
\begin{gathered}
\mu \lambda=\frac{\Delta^{\prime}}{\Delta}, \mu \lambda^{2}=\frac{\Delta^{\prime \prime}}{\Delta} \quad \text { e } \mu \lambda^{3}=\frac{\Delta^{\prime \prime}}{\Delta} \quad \text { e } \\
\lambda=\frac{\Delta^{\prime \prime}}{\Delta^{\prime \prime}}=\frac{\Delta^{\prime \prime}}{\Delta^{\prime}}
\end{gathered}
$$


A substituição de $\lambda$ em qualquer das equações primitivas fornece o valor de $\mu=\mathrm{Nm}$.

Os valores obtidos de $\mu$ e $\lambda$ conduzem, necessariamente, a um elipsóide pertencente ao conjunto infinito de elipsóides de $2 \mathrm{~V}$ igual ao do mineral ( $\cos 2 \mathrm{~V}=\mathrm{jj}$ ' $+\mathrm{mm}$ ' $+\mathrm{nn}$ '). Não se pode assegurar previamente, todavia, que seus semi-eixos sejam os índices de refração principais do mineral cuja lâmina está no microscópio.

\section{Determinação de $\lambda$ sem medição de birrefringência}

É possivel estabelecer uma expressão aproximada para o valor de $\lambda$, que teria a vantagem de prescindir de valores de birrefringência.

Com efeito, executada uma operação de extinção $(\varphi, \theta, \Psi)$, tem-se, no microscópio, uma secção de semi-eixos $\mathrm{N}_{1}$ e $\mathrm{N}_{2}$. A teoria do método estabelece:

$$
\mathrm{N}_{1}^{2}=\frac{\mu^{2}}{1-4 \lambda \alpha} \quad \text { e } \quad \mathrm{N}_{2}^{2}=\frac{\mu^{2}}{1-4 \lambda \beta}
$$

Desenvolvendo em série obtém-se:

$$
\begin{aligned}
& \mathrm{N}_{1}=\mu\left(1+2 \lambda \alpha+6 \lambda^{2} \alpha^{2}+20 \lambda^{3} \alpha^{3}+\ldots\right) \\
& \mathrm{N}_{2}=\mu\left(1+2 \lambda \beta+6 \lambda^{3} \beta^{2}+20 \lambda^{3} \beta^{3}+\ldots\right)
\end{aligned}
$$

Tendo em vista que $\lambda$ é pequeno (o elipsóide de índices de refração aproxima-se de uma esfera) podem ser negligenciadas as potências de $\lambda$ superiores a 1 . Assim:

$$
\mathrm{N}_{1}=\mu(1+2 \lambda \alpha) \text { e } \mathrm{N}_{2}=\mu(1+2 \lambda \beta)
$$

Elevando as expressões (3) ao quadrado. comparando com (1) e simplificando:

$$
\lambda=\frac{3\left(\beta^{2}-\alpha^{2}\right)}{4\left(\alpha^{3}-\beta^{3}\right)}
$$

É necessário, naturalmente, um estudo experimental para determinar a conveniência do emprego de (4). Caso as aproximações não conduzam a erros exagerados, a eliminação da birrefringência é um fator a ser considerado.

\section{DETERMINAÇÃO DE $B=\mathbf{N g}-\mathbf{N p}$}

A teoria do método estabelece que os três índices de refração principais são:

$$
\mathrm{N}_{1}=\frac{\mu^{2}}{1+4 \lambda \operatorname{sen}^{2} V}
$$




$$
\begin{aligned}
& \mathrm{N}_{2}=\mu=\mathrm{Nm} \\
& \mathrm{N}_{3}=\frac{\mu^{2}}{1-4 \lambda \cos ^{2} \mathrm{~V}}
\end{aligned}
$$

A birrefringência máxima é, pois, $N_{3}-N_{1}$ ou $-\left(N_{3}-N_{1}\right)$, quer seja $N_{1}=N p$ e $\mathrm{N}_{3}=\mathrm{Ng}$ ou $\mathrm{N}_{1}=\mathrm{Ng}$ e $\mathrm{N}_{3}=\mathrm{Np}$. Desenvolvendo em série (5) e (6):

$$
\begin{aligned}
& \mathrm{N}_{1}=\mu\left(1-2 \lambda \operatorname{sen}^{2} \mathrm{~V}+6 \lambda^{2} \operatorname{sen}^{4} \mathrm{~V}-\ldots\right) \\
& \mathrm{N}_{2}=\mu\left(1+2 \lambda \cos ^{2} \mathrm{~V}-6 \lambda^{2} \cos ^{4} \mathrm{~V}+\ldots\right)
\end{aligned}
$$

Negligenciando potências de $\lambda$ superiores a 1 :

$$
\begin{gathered}
\mathrm{N}_{1}=\mu\left(1-2 \lambda \operatorname{sen}^{2} \mathrm{~V}\right) \\
\mathrm{N}_{3}=\mu\left(1+2 \lambda \cos ^{2} \mathrm{~V}\right) \\
\mathrm{ou} \\
\frac{\mathrm{N}_{3}-\mathrm{N}_{1}}{2}=\mu \lambda
\end{gathered}
$$

Para se determinar a birrefringência máxima, basta, pois, determinar $\mu \lambda$. Essa determinação traz, no método, dupla vantagem: é metade da birrefringência máxima e tem o mesmo sinal que o mineral.

$\mathrm{O}$ valor de $\mu \lambda$ pode ser determinado de diferentes maneiras.

\section{Determinação de $\mu \lambda$ pela equação geral de extinção}

Como se viu em 3.1., $\mu \lambda$ pode ser determinado diretamente dos determinantes obtidos com duas ou mais equações (1).

Determinação de $\mu \lambda$ com qualquer operação $(\varphi, \theta, \Psi, r)$

$\mathrm{Na}$ equação (1), negligenciando as potências de $\lambda$ superiores a 1 :

$$
\mu \lambda=\frac{r}{2(\alpha-\beta)}
$$

É possível, pois, determinar $\mu \lambda$ com uma única operação $(\varphi, \theta, \Psi, r)$. No caso particular em que $\varphi=0, \theta=0$ e $\Psi=0$ :

$$
\alpha=\mathrm{j} . \mathrm{j}, \quad \text { e } \quad \beta=\mathrm{m} \cdot \mathrm{m}^{\prime} \quad \text { ou }
$$




$$
\mu \lambda=\frac{r}{2\left(\mathrm{ij}^{\prime}-\mathrm{mm}\right)} \text {. Basta. pois, determinar a birrefringência }
$$

da posição de extinção inicial. Observe-se que, além de ser a posição mais rigorosamente determinada, pode ser repetida em 4 posições no plano inicial, levando em conta os sinais de r. O programa para computador já fornece o valor de

$$
\mathrm{C}=\frac{1}{2\left(\mathrm{jj}^{\prime}-\mathrm{mm}\right)}=\frac{\mu \lambda}{\mathrm{r}}
$$

\section{DETERMINAÇÃO ANALITICA DE Np, Nm e Ng}

Calculado $\mu \lambda$ por qualquer via, tomemos valores arbitrários para $\mu=\mathrm{Nm}$. Observe-se que pode ser atribuída a $\mu$ qualquer valor, mas convém que se atribuam valores possiveis como índices de refração. É possível, então, calcular $\lambda$ de $\mu \lambda$ e $\mathrm{Np}$ e $\mathrm{Ng}$ das expressões (5) e (6). Determinam-se, assim, infinitos conjuntos $\mathrm{Np}$, $\mathrm{Nm}$ e $\mathrm{Ng}$, todos com o mesmo $2 \mathrm{~V}$, já fornecido pelo computador. Assim, tem-se as possibilidades: $\left(\mathrm{Np}_{1}\right.$, $\left.\mathrm{Nm}_{1}, \mathrm{Ng}_{1}\right),\left(\mathrm{Np}_{2}, \mathrm{Nm}_{2}, \mathrm{Ng} 2\right)$, etd., ou, de um modo geral, $\left(\mathrm{Np}_{\mathrm{x}}, \mathrm{Nm}_{\mathrm{x}}, \mathrm{Ng}_{\mathrm{x}}\right)$.

$\mathrm{O}$ critério para escolher o conjunto do mineral que se tem na lâmina é o valor de $\mu \lambda=B / 2$ : o mineral tem os índices de refração principais para os quais $N g_{x}-N_{p_{x}}=$ $2 \mu \lambda$.

\section{CONCLUSÕES}

O estudo teórico realizado sugere as seguintes conclusões:

A indicatriz ótica de um mineral, dado em secção delgada arbitrária na platina universal pode, teoricamente, ser inteiramente determinada, em posição e grandeza, por via analítica, sem envolver outras medições que não ângulos de extinção e birrefringência.

Recomenda-se um estudo experimental para pesquisar se as aproximações envolvidas tornam o procedimento exeqüivel na prática.

Devem ser pesquisadas a determinação dos índices de refração principais e o valor da birrefringência máxima.

\section{SUMMARY}

ANALYTICAL DETERMINATION OF THE OPTICAL INDICATRIX OF MINERALS

An arbitrary thin section of a given mineral is considered as examined on the universal stage. The theoretical possibility of determining the principal refraction 
indexes by analytical method from data obtained from extinction and birefringence measurements is studied. A mathematical demonstration is presented to show that it is theoretically possible to determine $\mathrm{Np}, \mathrm{Nm}$ and $\mathrm{Ng}$ and, independently, $\mathrm{B}=\mathrm{Ng} \cdot \mathrm{Np}$.

\section{LITERATURA CITADA}

ABRAHÃO, I.O., 1968. Contribuição ao estudo do método analítico de Chomard. Tese de Livre-Docência apresentada à ESALQ, $132 \mathrm{p}$.

ABRAHÃO, I.O. e GODOY, C.R.M., 1971. Solução para computador do método analítico de Chomard. Anais da ESALQ, vol. XXVIII:227-234.

CHOMARD, L., 1934. Théorie et pratique de la méthode Fédorov. Procedé classique et méthode analy tique général. Dunod, Paris. Annales des Mines, tomo V:153-218. 
